\title{
Antibiotic-Associated Diarrhea
}

National Cancer Institute

\section{Source}

National Cancer Institute. Antibiotic-Associated Diarrhea. NCI Thesaurus. Code C128350.

Diarrhea that is associated with current or recent antibiotic use. 